\title{
Self-dual Planar Hypergraphs and Exact Bond Percolation Thresholds
}

\author{
John C. Wierman* \\ Department of Applied Mathematics and Statistics \\ Johns Hopkins University \\ wierman@jhu.edu \\ Robert M. Ziff ${ }^{\dagger}$ \\ Michigan Center for Theoretical Physics and Department of Chemical Engineering \\ University of Michigan \\ rziff@umich.edu
}

Submitted: Oct 4, 2010; Accepted: Mar 6, 2011; Published: Mar 24, 2011

Mathematics Subject Classification: 05C65, 60K35, 82B43

\begin{abstract}
A generalized star-triangle transformation and a concept of triangle-duality have been introduced recently in the physics literature to predict exact percolation threshold values of several lattices. Conditions for the solution of bond percolation models are investigated, and an infinite class of lattice graphs for which exact bond percolation thresholds may be rigorously determined as the solution of a polynomial equation are identified. This class is naturally described in terms of hypergraphs, leading to definitions of planar hypergraphs and self-dual planar hypergraphs. There exist infinitely many self-dual planar 3-uniform hypergraphs, and, as a consequence, there exist infinitely many real numbers $a \in[0,1]$ for which there are infinitely many lattices that have bond percolation threshold equal to $a$.
\end{abstract}

\section{Introduction}

\subsection{Bond Percolation}

Percolation is a random model on infinite lattices, which serves as the simplest lattice model example of a process exhibiting a phase transition. Even so, it provides some ex-

\footnotetext{
*Research supported by the Acheson J. Duncan Fund for the Advancement of Research in Statistics at Johns Hopkins University, and by sabbatical funding from the Mittag-Leffler Institute of the Swedish Royal Academy of Sciences

${ }^{\dagger}$ Research supported by National Science Foundation Grant No. DMS-0553487
} 
tremely challenging problems. Its study provides intuition for more elaborate statistical mechanics models. Due to its focus on clustering and connectivity phenomena, it is applied widely to problems such as magnetism and conductivity in materials, the spread of epidemics, fluid flow in a random porous medium, and gelation in polymer systems. Percolation models are studied extensively in both the mathematical and scientific literature. See Bollobás and Riordan [2], Grimmett [7], Hughes [8], and Kesten [10] for a comprehensive discussion of mathematical percolation theory, Stauffer and Aharony [20] for a physical science perspective, and Sahimi [15] for engineering science applications.

The bond percolation model may be described as follows. Consider an infinite connected graph $G$. Each edge of $G$ is randomly declared to be open with probability $p$, and otherwise closed, independently of all other edges, where $0 \leq p \leq 1$. (Note that the Erdős-Renyi random graph model represents percolation on the complete graph.) The corresponding parameterized family of product measures on configurations of edges is denoted by $P_{p}$. For each vertex $v \in G$, let $C(v)$ be the open cluster containing $v$, i.e. the connected component of the subgraph of open edges in $G$ containing $v$. Let $|C(v)|$ denote the number of vertices in $C(v)$. The percolation threshold of the bond percolation model on $G$, denoted $p_{c}(G$ bond $)$, is the unique real number such that

$$
p>p_{c}(G \text { bond }) \Longrightarrow P_{p}(\exists v \text { such that }|C(v)|=\infty)>0
$$

and

$$
p<p_{c}(G \text { bond }) \Longrightarrow P_{p}(\exists v \text { such that }|C(v)|=\infty)=0 .
$$

For over fifty years since the origins of percolation theory by Broadbent and Hammersley [4], the derivation of percolation thresholds has been a challenging problem. Until recently, exact solutions had been proved only for arbitrary trees [11] and a small number of periodic two-dimensional graphs [9, 10, 21, 22]. These results were obtained using graph duality and a star-triangle transformation. Scullard [16] introduced a generalized star-triangle transformation which allowed prediction of the exact site percolation threshold for the so-called "martini" lattice. A triangle-triangle transformation and concept of triangle-duality was introduced by Ziff [31] and Chayes and Lei [5], and further developed by Ziff and Scullard [17, 32]. Triangle-duality allowed derivation of exact thresholds for an additional collection of "martini-like" lattices and other lattice graphs.

In this article, we introduce a mathematical framework for unifying the concepts developed in the previous research. We examine these new derivations and identify and explain conditions under which the results can be proved rigorously mathematically. For this purpose, we describe a class of lattices solvable for the bond percolation threshold, using the graph-theoretical concept of hypergraphs, and define planar hypergraphs and a concept of self-duality for them. We discuss replacing each hyperedge in a self-dual planar hypergraph by a planar graph called a "generator" to obtain a solvable lattice graph. For the proof that it is solvable, we construct a dual generator and dual lattice, and apply the generalized star-triangle transformation to derive the exact bond percolation threshold. Certain technical conditions, such as planarity and periodicity are used to complete a rigorous mathematical proof of the derivation. In section 9, we comment on the possible extension of the method to site models and nonplanar lattices. 

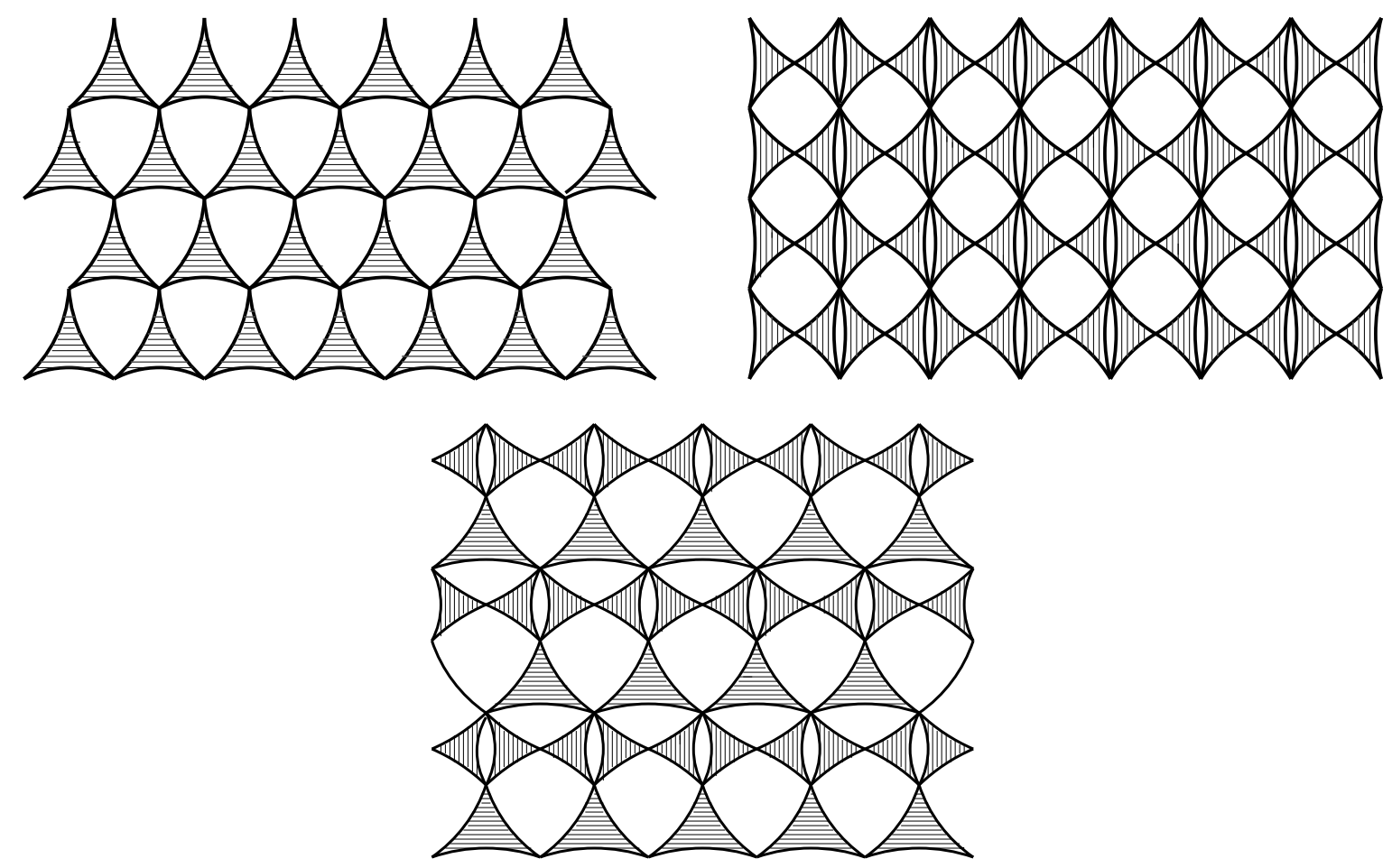

Figure 1: Self-dual hypergraph arrangements illustrated in [32]. In the top row, we refer to the left as the triangular arrangement, the right as the bow-tie arrangement. The third example alternates rows of triangles and bow-ties.

\subsection{The Triangle-Duality Construction}

We first briefly and loosely describe the triangle-duality approach, in the context of bond percolation, with a slightly different perspective: We consider constructing a lattice graph rather than decomposing one. Consider an arrangement of non-overlapping triangular regions in the plane, with triangles touching only at their vertices. For convenience, it is sometimes desirable to represent the triangles as slightly concave, as illustrated in Figure 1. Such an arrangement may be transformed into another arrangement via the "triangle-triangle transformation," in which each triangle is replaced by a "reversed triangle" as shown in Figure 2. If the resulting (dual) triangular arrangement is equivalent to the original arrangement, the arrangement is called "self-dual under the triangle-triangle transformation" by Ziff and Scullard. If the triangular arrangement is self-dual, then a lattice may be constructed by replacing each triangular region by a network of bonds which has vertices at all three vertices of the triangle. Such a network will be called the generator of the lattice. From such a generator, it may be possible to construct a dual generator, which creates another lattice when replacing the triangles in the dual triangular arrangement. By solving an equation derived from the connection probabilities in the generator and dual generator, a solution for the percolation threshold is obtained. 


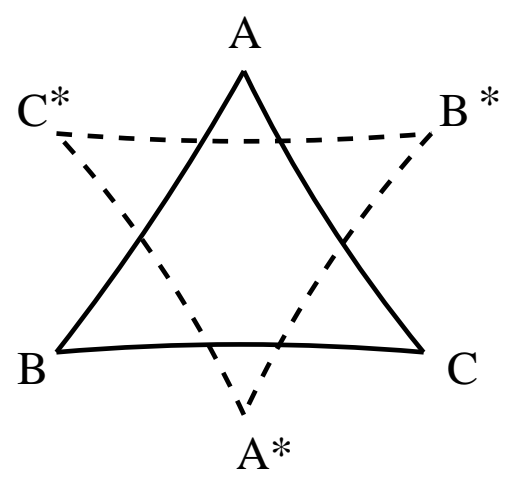

Figure 2: Solid lines represent a 3-hyperedge with boundary vertices $A, B$, and $C$. Dashed lines represent the "reversed" or dual hyperedge, with its boundary vertices $A^{*}, B^{*}$, and $C^{*}$ labeled in the proper positions.

One goal of this article is to make explicit some assumptions which may have been implicit in [16], [17], [31] and [32]. In the remainder of this article, we discuss conditions which allow a valid exact solution for the bond percolation threshold in the framework of 3-regular hypergraphs. Here we only note some remarks and cautions regarding a few issues. (1) Planarity and graph duality play crucial roles in our reasoning, as in all rigorous solutions for bond percolation thresholds of periodic lattices. Our results only directly apply to planar hypergraphs and planar generators. There is some evidence of wider applicability, which is being investigated. (2) Care must be taken when constructing the dual hypergraph, with the reversed triangles connected in a precise manner in order to create a proper dual structure. The reversed triangles need not be the same size or shape as the original triangles, but may need to be distorted instead of merely reversed. (3) To apply standard percolation theory results to prove that that solution is valid, the resulting lattice graph must be periodic. However, Markström and Wierman [12] have constructed examples of aperiodic models for which the bond percolation threshold is exactly determined, using a periodic hypergraph into which a rotor gadget used as generator and its reflection are placed in an aperiodic manner.

\subsection{Equality of Percolation Thresholds}

For lattices constructed by this method, the value of the bond percolation threshold is determined by equations describing the probabilities of connections within the generator. Therefore, using the same generator in multiple self-dual triangular arrangements produces multiple lattices with equal bond percolation thresholds. Ziff and Scullard [32](Figures 1 and 6) illustrate three different self-dual arrangements. In section 7, we show that there are infinitely many self-dual 3-uniform hypergraphs, so each generator satisfying the appropriate conditions will generate an infinite set of lattices with equal percolation thresholds. Previously, it was only known that there were infinitely many lattices with bond percolation threshold equal to one-half, since Wierman [29] provided a 
construction for infinitely many periodic self-dual lattices. We also construct a sequence of nested generators which must give unequal percolation thresholds, which implies that there are infinitely many values $a$ for which there are infinitely many lattices with bond percolation threshold equal to $a$. The result also holds for site percolation thresholds, by the bond-to-site transformation.

\section{Background and Definitions}

\subsection{3-Uniform Hypergraphs}

Given a set $V$ of vertices, a hyperedge $H$ is a subset of $V$. A hyperedge $H$ is said to be incident to each of its vertices. A $k$-hyperedge is a hyperedge containing exactly $k$ vertices. In order to neglect the detailed structure of our generators, at times we will view a generator as a 3-hyperedge, and will represent it in the plane as a shaded triangular region bounded by a slightly concave triangular boundary.

A hypergraph is a vertex set $V$ together with a set of hyperedges of vertices in $V$. A hypergraph containing only $k$-hyperedges is a $k$-uniform hypergraph. A hypergraph is planar if it can be embedded in the plane with each hyperedge represented by a bounded region enclosed by a simple closed curve with its vertices on the boundary, such that the intersection of two hyperedges is a set of vertices in $V$.

In order to construct lattice graphs with exactly solvable bond percolation models, we will consider infinite connected planar periodic 3-uniform hypergraphs. A planar hypergraph $H$ is periodic if there exists an embedding with a pair of basis vectors $\mathbf{u}$ and $\mathbf{v}$ such that $H$ is invariant under translation by any integer linear combination of $\mathbf{u}$ and $\mathbf{v}$, and such that every compact set of the plane is intersected by only finitely many hyperedges.

If a hypergraph $H$ is planar, we may construct a dual hypergraph $H^{*}$ as follows. Place a vertex of $H^{*}$ in each face of $H$. For each hyperedge $e$ of $H$, construct a hyperedge $e^{*}$ of $H^{*}$ consisting of the vertices in the faces surrounding $e$. Note that if the hyperedge is a 3-hyperedge represented by a triangular region, and each of the boundary vertices is in at least two hyperedges, then the dual hyperedge is a 3-hyperedge also, represented by a "reversed triangle."

Two hypergraphs are isomorphic if there is a one-to-one correspondence between their vertex sets which preserves all hyperedges. A hypergraph is self-dual if it is isomorphic to its dual. If the hypergraph is 3-uniform, this corresponds to the term triangle-dual used by Ziff and Scullard. To illustrate, in Figure 1 we provide three examples of infinite connected planar periodic self-dual 3-uniform hypergraphs mentioned in [32].

As a particular caution, note that the dual hypergraph is not obtained by simply reversing the triangles in the original hypergraph. The reversed triangles must be connected in a specific manner in order to create a proper dual structure. The way the reversed triangles are connected in the empty faces of the original structure is important. The reversed triangles do not need to be the same size or shape as the original triangles, but may need to be distorted instead of simply reversed. An example of a hypergraph 

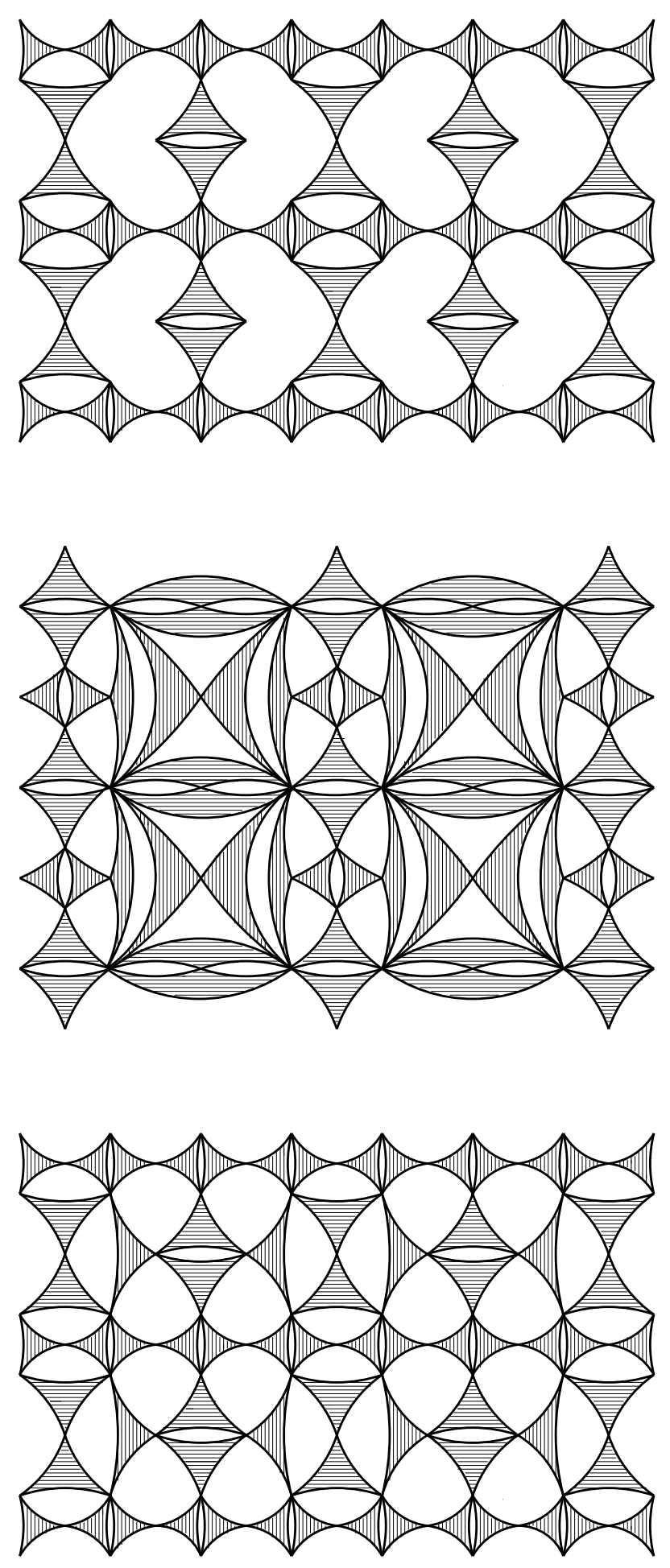

Figure 3: Top: A example of a hypergraph which is not self-dual, but appears to be if one simply reverses each triangle. Middle: The dual of the hypergraph above. Bottom: A selfdual hypergraph constructed by inserting additional 3-hyperedges in the top arrangement. 
which appears to be self-dual if one reverses each triangle, but is actually not self-dual, is given in Figure 3.

\section{$3 \quad$ Generators and Duality}

A planar graph can be embedded in the plane so that edges meet only at their endpoints, which divides the plane into regions bounded by edges, called "faces." If the planar graph is finite and connected, one of these faces is unbounded. A generator is a finite connected planar graph embedded in the plane so that three vertices on the unbounded face are designated as boundary vertices, which we denote as A, B, and C.

Given a generator $\mathrm{G}$, we construct a dual generator $G^{*}$ by placing a vertex in each bounded face of $G$, and three vertices $A^{*}, B^{*}$, and $C^{*}$ of $G^{*}$ in the unbounded face of $G$, as follows: The boundary of the unbounded face can be decomposed into three (possibly intersecting) paths, from $A$ to $B, B$ to $C$, and $C$ to $A$. The unbounded face may be partitioned into three unbounded regions by three non-intersecting polygonal lines starting from $A, B$, and $C$. Place $A^{*}$ in the region containing the boundary path connecting $B$ and $C, B^{*}$ in the region containing the boundary path connecting $A$ and $C$, and $C^{*}$ in the region containing the boundary path connecting $A$ and $B . A^{*}, B^{*}$, and $C^{*}$ are the boundary vertices of $G^{*}$.

For each edge $e$ of $G$, construct an edge $e^{*}$ of $G^{*}$ which crosses $e$ and connects the vertices in the faces on opposite sides of $e$. If $e$ is on the boundary of the infinite face, connect it to $A^{*}$ if $e$ is on the boundary path between $B$ and $C$, to $B^{*}$ if $e$ is between $A$ and $C$, and connect it to $C^{*}$ if $e$ is between $A$ and $B$. (Note that it is possible for $e^{*}$ to connect more than one of $A^{*}, B^{*}$, and $C^{*}$, for example, if there is a single edge incident to $A$ in $G$, so its dual edge connects $B^{*}$ and $C^{*}$.)

Note that $G^{*}$ is not the dual graph of $G$, which would have only one vertex in the unbounded face. The three vertices $A^{*}, B^{*}$, and $C^{*}$ will correspond to separate faces of the lattice $L_{G}$ generated from $G$.

Given a planar generator $G$ and a connected periodic self-dual 3-uniform hypergraph $\mathcal{H}$, a dual pair of periodic lattices may be constructed as follows: Construct a lattice graph $L_{G, \mathcal{H}}$ by replacing each hyperedge of $\mathcal{H}$ by a copy of the generator $G$, with the boundary vertices of the generator corresponding to the vertices of the hyperedge, in such a manner that the resulting lattice is periodic. This is always possible, by choosing the embeddings of the generator in one period of the hypergraph, and extending the choice periodically. (However, for a generator without sufficient symmetry, it may be possible to embed the generator in hyperedges in a way that produces a non-periodic lattice, so some care is needed.)

We now construct a lattice $L_{G^{*}, \mathcal{H}^{*}}$ as follows: Construct the embedding of the dual hypergraph $\mathcal{H}^{*}$ in the plane, in which every hyperedge of $\mathcal{H}$ is reversed. Replace each hyperedge of $\mathcal{H}^{*}$ by a copy of the dual generator $G^{*}$, embedded so that it is consistent with the embedding of $G$, that is, in all hyperedges boundary vertex $A^{*}$ in $G^{*}$ is opposite vertex $A$ in $G, B^{*}$ is opposite $B$, and $C^{*}$ is opposite $C$, and each edge of $G^{*}$ crosses the 
appropriate edge of $G$. This results in a simultaneous embedding of $L_{G^{*}, \mathcal{H}^{*}}$ and $L_{G, \mathcal{H}}$. An example of the construction for a particular generator is illustrated in Figure 4.

The constructions of the two lattices both produce a planar representation of the resulting lattice. From the simultaneous embeddings of the two lattices, it is seen that $L_{G^{*}, \mathcal{H}^{*}}$ is the dual lattice of $L_{G, \mathcal{H}}$, since there is a one-to-one correspondence between vertices of one and faces of the other, and a one-to-one correspondence between edges, which are paired by crossing. (Note that the position of the boundary vertices in $L_{G^{*}, \mathcal{H}^{*}}$ is completely determined by the positions of boundary vertices in $L_{G, \mathcal{H}}$. Rotations or reflections of the generator $G^{*}$ for any hyperedge may not produce a dual pair of lattices.)

\section{Reduction to a Single Equation}

Consider a generator $G$ and its dual generator $G^{*}$. In each case, denote the three boundary vertices by $A, B$, and $C$ listed counterclockwise around the triangle from the initial vertex. Any configuration (i.e., designation of edges or vertices as open or closed) on $G$ determines a partition of the boundary vertices into clusters of vertices that are connected by open edges. Each such "boundary partition" may be denoted by a sequence of vertices and vertical bars, where vertices are in distinct open clusters if and only if they are separated by a vertical bar. For example, $A B \mid C$ indicates that, within $G$, the vertices $A$ and $B$ are in the same open cluster, but $C$ is in a separate cluster.

Given a planar embedding of the lattice $L_{G, \mathcal{H}}$ and a planar embedding of $L_{G^{*}, \mathcal{H}^{*}}$ with each edge crossing its dual edge, we may define coupled percolation models. Let each edge of $L_{G, \mathcal{H}}$ be open with probability $p$ independently of all other edges, and define each edge of $L_{G^{*}, \mathcal{H}^{*}}$ to be open if and only if its dual edge is open.

Suppose we have two bond percolation models on $L_{G, \mathcal{H}}$ and $L_{G^{*}, \mathcal{H}^{*}}$, with different edge probability parameters $p$ and $q$, each assigning probability to configurations on $G$ and $G^{*}$, respectively. The probability, denoted $P_{p}^{G}(\pi)$ or $P_{q}^{G^{*}}(\pi)$ respectively, for the partition $\pi$ is determined by summing the probabilities of all configurations that produce the partition $\pi$ of the boundary vertices.

The set of boundary partitions is a partially ordered set (poset). A partition $\pi$ is a refinement of $\sigma$, denoted $\pi \leq \sigma$, if every cluster of $\pi$ is contained entirely in a cluster of $\sigma$. The set of boundary partitions ordered by refinement is a combinatorial lattice, called the partition lattice.

Thus, we have two probability measures, $P_{p}^{G}$ and $P_{q}^{G^{*}}$ on the partition lattice, which summarize probabilities of connections between the boundary vertices without explicitly referring to the detailed structure of the generator and its dual. The remarkable fact that allows exact bond percolation threshold values to be obtained is that it is possible to choose the parameters $p$ and $q$ so that the two probability measures are exactly equal. (Note that in cases with more boundary vertices, where the probability measures cannot be made equal, the concept of stochastic ordering of probability measures may be used to determine mathematically rigorous bounds for percolation thresholds, using the substitution method $[13,14,23,25,26,27,28]$. 

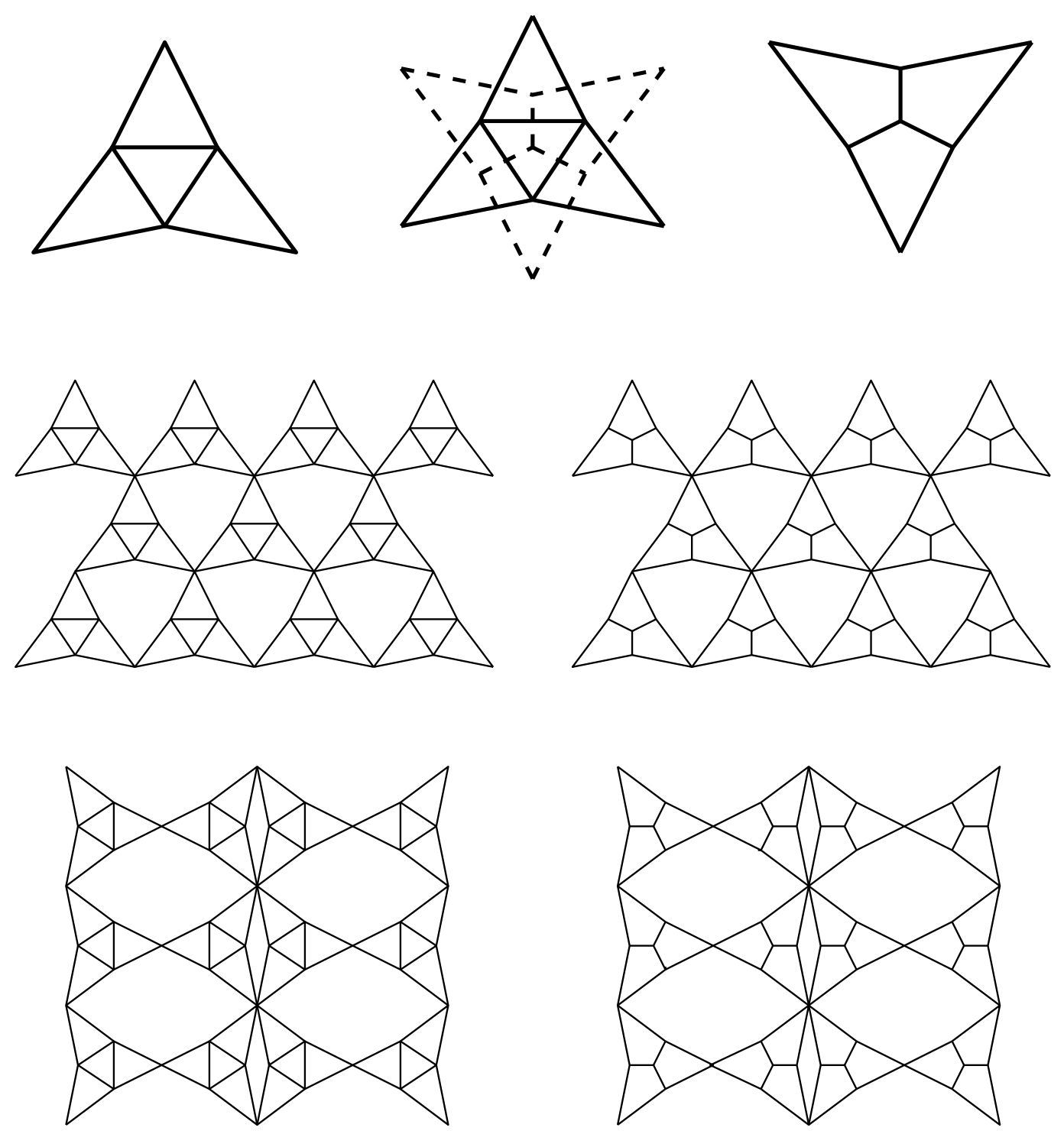

Figure 4: The construction of lattices based on a specific generator. Top: The generator, the duality relationship, and the dual generator. Middle: The lattices based on the generator and the triangular hypergraph arrangement. Bottom: The lattices based on the generator and the bow-tie hypergraph arrangement. 
By the duality relationship between $G$ and $G^{*}$, we have that for each configuration of open and closed edges, the following five statements hold:

1. $A, B$, and $C$ are connected by open paths if and only if $A^{*}, B^{*}$, and $C^{*}$ are in separate closed components.

2. $A$ and $B$ are connected by an open path, but $C$ is in a separate open component if and only if $A^{*}$ and $B^{*}$ are connected by a closed path, but $C^{*}$ is in a separate closed component.

3. $A$ and $C$ are connected by an open path, but $B$ is in a separate closed component, if and only if $A^{*}$ and $C^{*}$ are connected by a closed path, but $B^{*}$ is in a separate closed component.

4. $B$ and $C$ are connected by an open path, but $A$ is in a separate closed component, if and only if $B^{*}$ and $C^{*}$ are connected by a closed path, but $A^{*}$ is in a separate closed component.

5. $A, B$, and $C$ are in separate open components if and only if $A^{*}, B^{*}$, and $C^{*}$ are connected by closed paths.

While these statements are intuitively clear by drawing diagrams, the proofs of these statements rely on duality. However, since the dual generator is not the dual graph of the generator, some additional vertices and edges must be added to apply graph duality results. Examples of such reasoning are given in Smythe and Wierman [19, pp. 8-9] and Bollobás and Riordan [2, pp.55-56].

When considering the random configurations induced by a percolation model, the five statements become statements of equality of events, which then have equal probabilities, yielding

$$
\begin{aligned}
& P_{p}^{G}[A B C]=P_{1-p}^{G^{*}}\left[A^{*}\left|B^{*}\right| C^{*}\right], \\
& P_{p}^{G}[A B \mid C]=P_{1-p}^{G^{*}}\left[A^{*} B^{*} \mid C^{*}\right], \\
& P_{p}^{G}[A C \mid B]=P_{1-p}^{G^{*}}\left[A^{*} C^{*} \mid B^{*}\right], \\
& P_{p}^{G}[A \mid B C]=P_{1-p}^{G^{*}}\left[A^{*} \mid B^{*} C^{*}\right], \\
& P_{p}^{G}[A|B| C]=P_{1-p}^{G^{*}}\left[A^{*} B^{*} C^{*}\right] .
\end{aligned}
$$

Since $p$ is still a free parameter, we may choose it to satisfy

$$
P_{p}^{G}[A B C]=P_{1-p}^{G^{*}}\left[A^{*} B^{*} C^{*}\right]
$$

This equation always has a solution in $[0,1]$ since the left side is an increasing polynomial function of $p$ while the right side is decreasing polynomial, both with values varying between 0 and 1 . With this choice of $p$, the four probabilities in the first and last equations 
are equal, and, in fact, the two probability measures are equal. Thus, it is equivalent to solve the connectivity equation

$$
P_{p}^{G}[A B C]=P_{p}^{G}[A|B| C],
$$

equating the probabilities of all boundary vertices connected and no boundary vertices connected in the generator of the lattice. In the following, we denote the solution to this polynomial equation by $p_{0}$.

\section{Exact Solution for the Bond Percolation Threshold}

We now verify that the solution of equation (9) provides the exact value of the bond percolation threshold. We state the result as the following theorem.

Theorem: Let $G$ be a connected planar generator with three boundary vertices $A, B$, and $C$, and let $\mathcal{H}$ be a connected periodic self-dual planar 3-uniform hypergraph. Suppose that the lattice $L_{G, \mathcal{H}}$ is periodic with (at least) one axis of reflection symmetry. Then the bond percolation thresholds of $L_{G, \mathcal{H}}$ and $L_{G^{*}, \mathcal{H}^{*}}$ satisfy

$$
p_{c}\left(L_{G, \mathcal{H}}\right)=p_{0}=1-p_{c}\left(L_{G^{*}, \mathcal{H}^{*}}\right)
$$

where $p_{0}$ is the solution in $[0,1]$ of the polynomial equation

$$
P_{p}^{G}(A B C)=P_{p}^{G}(A|B| C) .
$$

Proof Sketch: We rely on relatively standard results in mathematical percolation theory. Kesten [10] proved that for a dual pair of periodic planar lattices, $L$ and $L^{*}$, with at least one axis of reflection symmetry,

$$
p_{c}(L)+p_{c}\left(L^{*}\right)=1
$$

and that various definitions of the percolation threshold, denoted by $p_{H}, p_{T}$, and $p_{S}$ are all equal for each lattice. (The result was actually stated for the more general setting of site models, and applies to bond models by first applying the bond-to-site transformation to obtain an equivalent site model.) To apply Kesten's result to lattices constructed by the method in this article, we use the $p_{S}$ concept of the percolation threshold.

For a periodic graph, the percolation threshold $p_{S}$ is defined in terms of the asymptotic behavior of probabilities that open clusters connect opposite sides of rectangles in sequences of similar rectangles whose areas are increasing to infinity. In the following, we give a brief general description which can be adapted to each of the lattices in our construction, but avoiding some technicalities that are specific to each lattice.

Now let $L=L_{G, \mathcal{H}}$ be a lattice constructed in this article, and let $L^{*}=L_{G^{*}, \mathcal{H}^{*}}$ denote its dual lattice. $L$ may be embedded periodically in the plane such that the unit vectors in the $x$ - and $y$-axis directions are the basis vectors and the $x$-axis is an axis of symmetry. 
For each pair of positive integers, $m$ and $n$, let $L_{m \times n}$ denote the graph containing all edges of hyperedges of $L$ that intersect the region $[0, m] \times[0, n]$. We can specify sets of vertices of $L_{m \times n}$ which we call its top, bottom, left, and right sides. We are interested in the event that there is an open path which crosses $L_{m \times n}$ from left to right, or top to bottom, which we denote by $\left\{\right.$ open $\left.\leftrightarrow L_{m \times n}\right\}$, or $\left\{\right.$ open $\left.\uparrow L_{m \times n}\right\}$, respectively. Similarly, we consider also closed crossings. The percolation threshold $p_{S}$ is defined as

$$
p_{S}=\sup \left\{p: \lim _{n \rightarrow \infty} P_{p}\left[\text { open } \uparrow L_{3 n \times n}\right]=0 \text { and } \lim _{n \rightarrow \infty} P_{p}\left[\text { open } \leftrightarrow L_{n \times 3 n}\right]=0\right\} .
$$

In the following, we will use square regions, noting that if $\limsup _{n \rightarrow \infty} P_{p}[$ open $\leftrightarrow$ $\left.L_{n \times n}\right]>0$, then $\limsup _{n \rightarrow \infty} P_{p}\left[\right.$ open $\left.\leftrightarrow L_{n \times 3 n}\right]>0$, and similarly for vertical crossings. In either case, $p>p_{S}$.

Now let $L_{n \times m}^{*}$ denote the graph corresponding to the dual hyperedges of the hyperedges in $L_{n \times m}$. For $L_{n \times m}^{*}$, we can also specify sets of vertices of the lattices which we can call the top, bottom, left, and right sides of these regions, and define similar crossing events, in such a manner that duality yields

$$
P_{p_{0}}\left[\text { open } \leftrightarrow \text { in } L_{n \times n}\right]+P_{p_{0}}\left[\text { closed } \uparrow \text { in } L_{n \times n}^{*}\right]=1,
$$

which implies that

$$
P_{p_{0}}\left[\text { open } \leftrightarrow \text { in } L_{n \times n}\right]+P_{1-p_{0}}\left[\text { open } \uparrow \text { in } L_{n \times n}^{*}\right]=1 .
$$

We now replace generators of $L^{*}$ by generators of $L$. In the duality relationship, each hyperedge in $L^{*}$ is obtained by rotating the corresponding hyperedge of $L$ by $180^{\circ}$ degrees, so this replacement results in a copy of $L$ reflected through some horizontal line. However, the subgraph of $L$ corresponding to the replacement of hyperedges in $L_{n \times n}^{*}$ may not be isomorphic to $L_{n \times n}$. For convenience, the following argument is written as if it is isomorphic. (If not, by monotonicity, we may consider a rectangle which is slightly smaller vertically and slightly larger horizontally, which is sufficient to obtain the conclusion.)

While replacing the hyperedges, by using the equality of connectivities at parameters $p_{0}$ in $L$ and $1-p_{0}$ in $L^{*}$, we have

$$
P_{p_{0}}\left[\text { open } \leftrightarrow \text { in } L_{n \times n}\right]+P_{p_{0}}\left[\text { open } \uparrow \text { in } L_{n \times n}\right]=1 .
$$

As a consequence, either

$$
\limsup _{n \rightarrow \infty} P_{p_{0}}\left[\text { open } \leftrightarrow \text { in } L_{n \times n}\right] \geq \frac{1}{2}
$$

or

$$
\limsup _{n \rightarrow \infty} P_{p_{0}}\left[\text { open } \uparrow \text { in } L_{n \times n}\right] \geq \frac{1}{2},
$$

(or both) so

$$
p_{S}(L) \leq p_{0} .
$$


Reversing the roles of $L$ and $L^{*}$, we also obtain

$$
p_{S}\left(L^{*}\right) \leq 1-p_{0}
$$

Therefore,

$$
1 \leq p_{c}(L)+p_{c}\left(L^{*}\right)=p_{S}(L)+p_{S}\left(L^{*}\right) \leq p_{0}+\left(1-p_{0}\right)=1,
$$

so the percolation thresholds must equal their upper bounds, yielding

$$
p_{c}(L)=p_{0}
$$

and

$$
p_{c}\left(L^{*}\right)=1-p_{0} .
$$

\section{Subdivided and Split Edges}

There are two situations which can produce lattices which do not occur in natural physical models. If the generator has a boundary vertex with only one incident edge, it is possible for the resulting lattice to have two edges in series. If the generator has an edge which is incident to two boundary vertices, it is possible for the resulting lattice to have double edges, i.e., two edges between the same pair of vertices.

These problems cannot occur for some self-dual hypergraphs: In any hypergraph for which each boundary vertex is shared by three or more hyperedges, such as the triangular lattice arrangement, each boundary vertex has at least three incident edges, and thus there are no edges in series. In any hypergraph in which any two hyperedges intersect in at most one boundary vertex, such as the triangular arrangement, there cannot be parallel edges.

The generators illustrated in Figures 4 and 8 do not produce subdivided or split edges in any self-dual hypergraph arrangement. However, for other hypergraph arrangements, such as the bow-tie hypergraph, there can be subdivided edges and split edges. If all hyperedges have the same number of pendant boundary vertices involved in series edges and the same number of adjacent boundary vertices involved in parallel edges, in a periodic pattern, then the lattice can be transformed into a lattice without series or parallel edges for which the bond percolation threshold can be exactly determined: (1) If two hyperedges both have pendant vertices at the same boundary vertex, then consider each to have

probability $\sqrt{p}$ of being open, which is equivalent to one edge with probability $p$. (2) If two hyperedges have two boundary vertices in common and in both hyperedges these boundary vertices are adjacent, then to get one edge with probability $p$, consider each edge to be open with probability $s$ which satisfies $p=s^{2}+2 s(1-s)=1-(1-s)^{2}$, which implies that $s=1-\sqrt{1-p}$. Note that in both cases these edge probability functions are increasing functions of the parameter $p$, which is sufficient for the reasoning of this article to apply. 


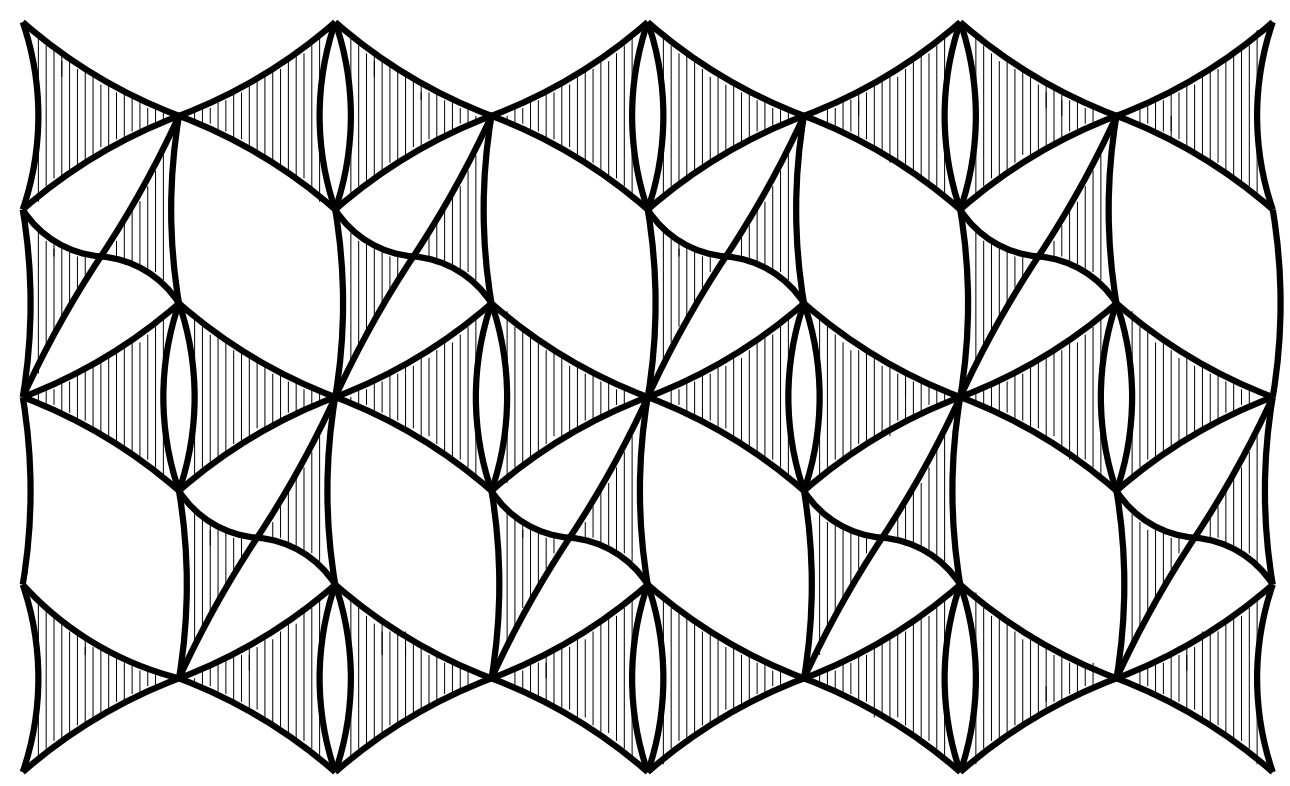

Figure 5: A self-dual hypergraph.

\section{Construction of Infinitely Many Non-Isomorphic Self-Dual Planar Hypergraphs}

In section 4, Ziff and Scullard [32] illustrate a third self-dual hypergraph, in addition to the triangular lattice structure and bow-tie lattice structure. We provide another self-dual hypergraph, which has not appeared previously, in Figure 5.

Ziff and Scullard [32] mention that many other self-dual arrangements can be constructed. In this section, we make make this comment more precise, by showing that there are in fact infinitely many self-dual hypergraphs.

Note that the bond percolation threshold of a graph constructed from a generator and a self-dual hypergraph is uniquely determined by the connectivity equation. By filling all self-dual 3-uniform hypergraphs with the same generator, we construct infinitely many planar lattices which have the same value for the bond percolation threshold.

We may construct self-dual hypergraphs using rows of similarly-oriented triangles and rows of similarly-oriented bow-ties, as shown in Figure 6. To form an infinite periodic selfdual hypergraph, we combine these into a periodic pattern. Consider forming a sequence of rows which alternates between $m$ rows of triangles and $n$ rows of bow-ties, for any $m \geq 1$ and $n \geq 1$. When constructing the dual hypergraph, each triangle is reflected 180 degrees, while the row of bow-ties is translated so the boundary vertices match up with the triangle vertices. As a result, the dual hypergraph is isomorphic to the original hypergraph reflected and translated. See Figure 6 for an illustration.

We may construct another infinite collection of self-dual hypergraphs similarly. At the top of Figure 7, we show a row of triangles with the top of every third triangle moved to the right. Each time a top vertex is moved to the right, it produces two hyperedges which 

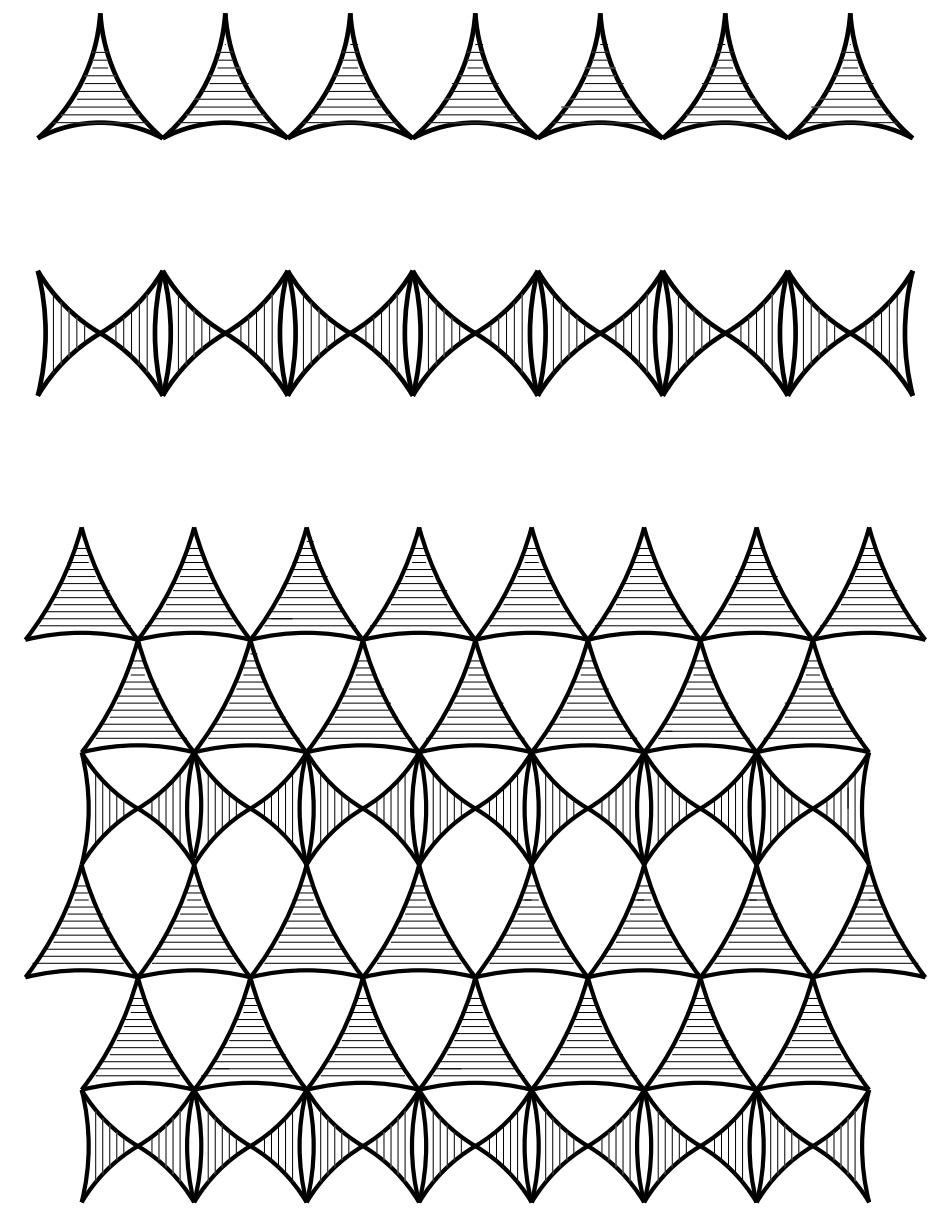

Figure 6: Top: A row of upward-pointing triangles. Middle: A row of bow-ties. Bottom: A periodic self-dual 3-regular hypergraph which alternates one row of bow-ties with two rows of triangles. 
share two boundary vertices, as occurs in the bow-tie lattice structure. Consider forming a sequence of rows which alternates between $m$ rows of triangles and $n$ rows of triangles with tops moved, in a periodic manner. Again, the dual hypergraph is isomorphic to the original hypergraph reflected and translated. See Figure 7, which illustrates this construction. Note that an arrangement in which all rows have every other top moved, the hypergraph is isomorphic to the bow-tie hypergraph.
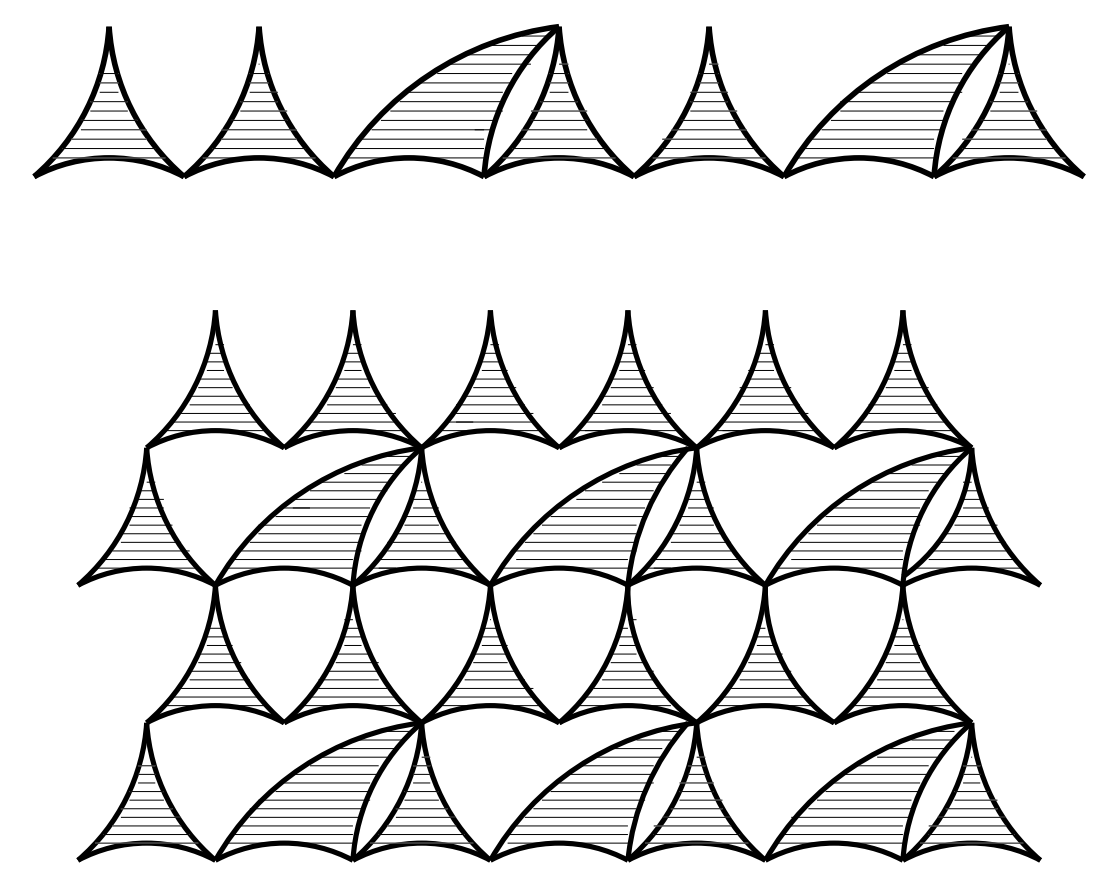

Figure 7: Top: A row of triangles with every third top moved to the right. Bottom: A self-dual hypergraph constructed by alternating rows of triangles and rows of triangles with every other top moved to the right.

\section{Infinitely-Many Threshold Values}

Figure 8 shows a sequence of generator graphs that are nested so that each graph contains every previous graph as a subgraph. Each generator can be embedded in infinitely many self-dual hypergraphs, producing infinitely many lattices which have the same bond percolation threshold. (Note that the construction does not have single edges connecting the boundary vertices, in order to avoid complications such as the lattice or its dual lattice having split edges or subdivided edges.) By the strict subgraph relationships between the generators, a result of Aizenmann and Grimmett [1] shows that the percolation thresholds of the families of graphs for different generators are not equal. Therefore, we have established that for infinitely many real numbers $a$ there are infinitely many periodic lattice graphs with bond percolation threshold equal to $a$. 

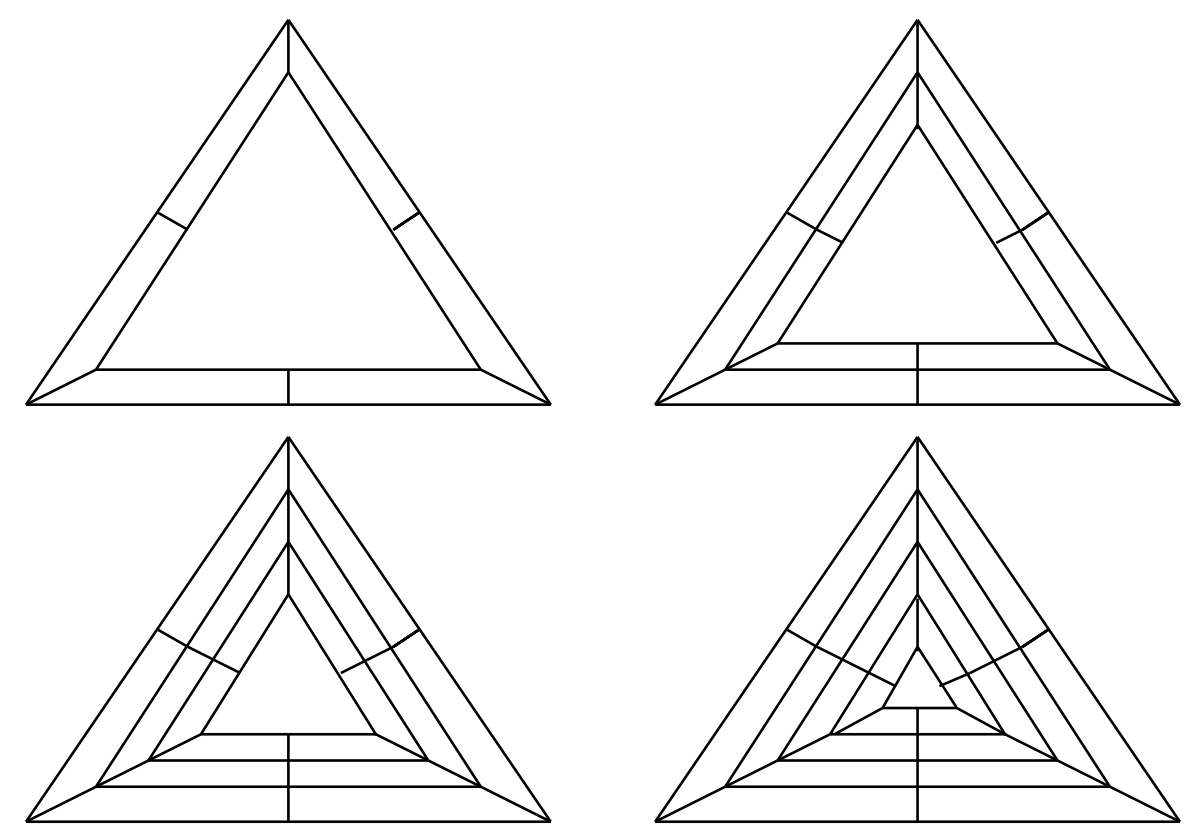

Figure 8: The first four graphs in an infinitely sequence of nested generators. Each contains all previous graphs in the sequence as subgraphs.

Recall that by Fisher's [6] bond-to-site transformation, the bond percolation model on a lattice $L$ is equivalent to the site percolation model on the covering lattice (in the physics literature) or line graph (in the mathematical literature), so that their percolation thresholds are equal. [Note that the result of the bond-to-site transformation is not always planar, so two-dimensional site models use the concept of "matching lattice" rather than dual lattice.] Thus, we have also shown that for infinitely many values $a$ there are infinitely many site percolation models with percolation threshold equal to $a$.

\section{Concluding Remarks}

We have provided sufficient conditions on the underlying lattice for correct application of the methods of Ziff and Scullard for determining exact bond percolation thresholds. The method applies if the lattice is constructed from an infinite connected planar periodic selfdual 3-regular hypergraph with one axis of symmetry, using a generator which is a finite connected planar graph with three boundary vertices. These conditions are naturally stated in terms of planar hypergraphs, reflect the need for planarity of the lattice in order to apply graph duality results, and reflect the need for technical conditions for validity of the mathematical proofs.

In particular, note that sufficient conditions reflect the current state of knowledge in mathematical percolation theory, requiring periodicity and an axis of symmetry. It is plausible that the results may be valid in a larger context, where these conditions are relaxed. As evidence for this, in a recent breakthrough, Bollobás and Riordan [2,3] exactly 
determined the site percolation threshold in a certain continuum percolation model which is not periodic or symmetric.

Ziff and Scullard also apply their approach to site percolation models and correlated percolation models (either all open or all closed). The formalism allows correlated bonds within each generator. As in the case of bond percolation, care must be taken to understand the conditions on the generator and the connections between generators that produce a valid solution. Research on these issues is in progress.

Wierman [24] used the so-called "substitution method" with the star-triangle transformation to show that, if they exist, the critical exponents are equal for the triangular and hexagonal lattice bond percolation models, and that they are equal for the bond percolation models on the bow-tie and its dual lattice. Sedlock and Wierman [18] generalized this result to the class of lattices identified in this article.

\section{References}

[1] Aizenman, M and Grimmett, G R (1991) Strict monotonicity for critical points in percolation and ferromagnetic models. J. Stat. Phys. 63, 817-835.

[2] Bollobás, B and Riordan, O (2006). Percolation. Cambridge University Press, Cambridge.

[3] Bollobás, B and Riordan, O (2006) The critical probability for random Voronoi percolation in the plane is 1/2. Probabability Theory and Related Fields 136, 417-468.

[4] Broadbent, S R and Hammersley, J M (1957) Percolation processes. I. Crystals and mazes. Proc. Camb. Phil. Soc. 53, 629-641.

[5] Chayes, L and Lei, H K (2006) Random cluster models on the triangular lattice. J. Stat. Phys. 122, 647-670.

[6] Fisher, M E (1961) Critical probabilities for cluster size and percolation problems. J. Math. Phys. 2, 620-627.

[7] Grimmett, G R (1999). Percolation. Springer.

[8] Hughes, B (1996). Random Walks and Random Environments. Volume 2: Random Environments. Clarendon Press, Oxford.

[9] Kesten, H (1980) The critical probability of bond percolation on the square lattice equals 1/2. Comm. Math. Phys. 74, 41-59.

[10] Kesten, H (1982) Percolation Theory for Mathematicians. Birkhäuser, Boston.

[11] Lyons, R (1990) Random walks and percolation on tree. Ann. Probab. 18, 931-958.

[12] Klas Markström and John C. Wierman (2010) Aperiodic non-isomorphic lattices with equivalent percolation and random-cluster models. Electronic Journal of Combinatorics, 17, R48.

[13] May, W D and Wierman, J C (2003) Recent improvements to the substitution method for bounding percolation thresholds. Congressus Numerantium 162, 5-25. 
[14] May, W D and Wierman, J C (2005) Using symmetry to improve percolation threshold bounds. Combinatorics, Probabability and Computing 14, 549-566.

[15] Sahimi, M (1994) Applications of Percolation Theory. Taylor \& Francis.

[16] Scullard, C R (2006) Exact site percolation thresholds using a site-to-bond transformation and the star-triangle transformation. Phys. Rev. E 73, 016107.

[17] Scullard, C R and Ziff, R M (2006) Predictions of bond percolation thresholds for the kagomé and Archimedean $\left(3,12^{2}\right)$ lattices. Phys. Rev. E 73, 045102(R)

[18] Sedlock, M R A and Wierman, J C (2009) Equality of bond-percolation critical exponents for pairs of dual lattices. Physical Review E 79, 05119.

[19] Smythe, R T and Wierman, J C (1978) First-Passage Percolation on the Square Lattice. (Lecture Notes in Mathematics, 671.) Springer, Berlin.

[20] Stauffer, D and Aharony, A (1991) Introduction to Percolation Theory. Taylor \& Francis.

[21] Wierman, J C (1981) Bond percolation on the honeycomb and triangular lattices. Adv. Appl. Probab. 13, 298-313.

[22] Wierman, J C (1984) A bond percolation critical probability determination based on the star-triangle transformation. J. Phys. A: Math. Gen. 17, 1525-1530.

[23] Wierman, J C (1990) Bond percolation critical probability bounds for the Kagome lattice by a substitution method. Disorder in Physical Systems, Oxford University Press, 349-360.

[24] Wierman, J C (1992) Equality of the bond percolation critical exponents for two pairs of dual lattices, Combinatorics, Probab. Comput. 1, 95-105.

[25] Wierman, J C (1995) Substitution method critical probability bounds for the square lattice site percolation model. Combinatorics, Probability and Computing, 4, 181188.

[26] Wierman, J C (2001) Site percolation critical probability bounds for the $\left(4,8^{2}\right)$ and $(4,6,12)$ lattices. Congressus Numerantium 150, 117-128.

[27] Wierman, J C (2002) Bond percolation critical probability bounds for three Archimedean lattices. Rand. Struct. \& Alg. 20, 507-518.

[28] Wierman, J C (2002) An improved upper bound for the hexagonal lattice site percolation critical probability. Combinatorics, Probab. Comput. 11, 629-643.

[29] Wierman, J C (2006) Construction of infinite self-dual graphs. Proc. 5th Hawaii International Conference on Statistics, Mathematics and Related Fields, [CD-ROM]

[30] Wu, F Y (2006) New critical frontiers for the Potts and percolation models. Physical Review Letters 96, 090602.

[31] Ziff, R M (2006) Generalized cell-dual-cell transformation and exact thresholds for percolation. Phys. Rev. E 73, 016134.

[32] Ziff, R M and Scullard, C R (2006) Exact bond percolation thresholds in two dimensions. J. Phys. A: Math. Gen. 39, 15083-15090. 